Theories \& Applications, the International Edition

Printed Version : (ISSN 2090-5262)

Online Version : (ISSN 2090-5270)

November 2014, Volume 4, No. 3 Pages (40 - 46)

\title{
Body Components and Their Relationship with Skill Performance within Top Level Wrestlers.
}

\section{Dr. Waleed Mohsen Moustafa Nasra}

Lecturer, Combat and individual sports department - Faculty of Physical Education for Men, Alexandria University, Egypt.

\begin{abstract}
This research general aim could be summarized in researcher is trying to present the most important relationships between body components and skills performances, which occur within wrestlers through training and competition and make it a scientific basis for the selection and training process.
\end{abstract}

In light of this object, wrestling specific body components availability is necessary to develop and improve wrestler physical state, while specific physical abilities availability is important to improve the skills performance and both are necessary to contribute into training process completion and access sports form during competition with lowest physical effort with the ability for fast recovery; as body components are better as physical are high, hence skills performance is higher.

Therefore, Researcher believes that it should be relied on physical components and using scientific methods based on measurement and application of specific tests for physical and skills abilities, which aims to good preparation and help to raise level of skills performance; then after achieve top level of sports activities generally and wrestling especially.

To access top sports level which achieve excellence, wrestling training programs should be planned to help acquire body components which are appropriate for wrestling that is to be done through body components measurements, which in turn affect physical abilities and then improved skills performance, and follow scientific steps to plan training programs that achieve global level aim.

Research results proof that there is correlation between wrestlers' skills performance and body components.

\section{Introduction:}

$\mathrm{S}$ kills performance and body measurements are of the most important components that must be taken into account to achieve champion level, fat and muscle tissue percentage have a close relationship with all other physical fitness components where each affects the other, for example, Fat increase negatively affect some physical fitness components like aerobic and anaerobic abilities and flexibility, as well as muscle tissue increase affect positively muscular strength and endurance, and speed, body composition is very important when sport activity requires vertical or horizontal body movement in the space. (AbulEla Abdel Fattah and Mohamed Sobhy Hassanein, 1997: p346) (Hussein Ahmed Heshmat, 1999:p75)

Determining sports fit body components has become a key goal for many coaches when designing physical preparation programs, which aims to develop different physical abilities, and then upgrading skill and tactical aspects in all sports activities. Each sport activity has its own body and physiological requirements; which should be available to those who target medals and championships in this activity. (Janet L. Walberg, 1999:p293)

Wrestling is one of the sports activities that require wrestler to have many of body components and special physical abilities, according to the special nature of the combat, which makes wrestler skills performance reflects his proper possession of those capabilities.
Wrestling specific body components availability is necessary to develop and improve wrestler physical state, while specific physical abilities availability is important to improve the skills performance and both are necessary to contribute into training process completion and access sports form during competition with lowest physical effort with the ability for fast recovery; as body components are better as physical are high, hence skills performance is higher.

Therefore, Researcher believes that it should be relied on physical components and using scientific methods based on measurement and application of specific tests for physical and skills abilities, which aims to good preparation and help to raise level of skills performance; then after achieve top level of sports activities generally and wrestling especially.

To access top sports level which achieve excellence, wrestling training programs should be planned to help acquire body components which are appropriate for wrestling that is to be done through body components measurements, which in turn affect physical abilities and then improved skills performance, and follow scientific steps to plan training programs that achieve global level aim.

Horswill (2008, p25), and Oppliger et. al. (2008, p156) are in the opinion that determining physical adaptations characteristics for wrestlers performance is the basis for success in combat, training loads tune, and training process organization, which is imperative matter in detecting those physical abilities and body components within top wrestlers as they are considered as national treasure. 
Since top level wrestlers are the in wrestlers field, so it was necessary to identify the relationship between their body components and sports performance, which in turn contributes to upgrade their level; and then realize more international championships and achievements .

Based on the above the scientific importance, which can be added by this study from information about top level wrestlers' body components characteristics and their relationship with skills performance, as it considered a scientific basis to select appropriate athletes for wrestling, standardize training loads, and an important indicator for wrestling training programs organizing and directing, and comparing local and global levels.

Through this study, researcher is trying to present the most important relationships between body components and skills performances, which occur within wrestlers through training and competition and make it a scientific basis for the selection and training process.

\section{Research objectives:}

\section{This research aims to identify:}

1- Body components of top level wrestlers.

2- Skills performance of top level wrestlers.

3- The relation between body components and skills performance within wrestlers.

\section{Research hypothesis}

In the light of research objectives research hypothesis was worded as follows:

- There is a relationship between body components and skill performance level within wrestlers.

\section{Research procedures:}

\section{I-Research Methodology:}

The researcher used the descriptive approach as to appropriate to research nature and objective.

\section{II- Research domains:-}

\section{Human domain:}

\section{Research sample:}

The research sample was chosen intentionally and consisted from (28) wrestlers from Egyptian youth wrestling national team, and wrestlers hold first positions in Alexandria clubs, as Alexandria is one of wrestling castles in Egypt.

\section{Study sample characteristics:}

Table (1)

arithmetic mean, standard deviation and skewness coefficient of wrestlers

\begin{tabular}{|c|c|c|c|c|}
\hline No. & $\begin{array}{r}\text { Statistical } \\
\text { significance } \\
\text { components }\end{array}$ & Mean & $\begin{array}{l}\text { Standard } \\
\text { deviation }\end{array}$ & $\begin{array}{l}\text { Skewness } \\
\text { coefficient }\end{array}$ \\
\hline 1 & Age (years) & 19.27 & 0.36 & 0.08 \\
\hline 2 & Height (cm ) & 175.07 & 6.098 & -0.07 \\
\hline 3 & Weight ( kg ) & 80.07 & 16.21 & 0.37 \\
\hline 4 & Body mass index ( \% ) & 25.34 & 3.48 & 1.78 \\
\hline 5 & Total Body Water (TBW) (kg) & 49.83 & 8.03 & 0.23 \\
\hline 6 & Fat -free mass (FFM) ( kg ) & 69.97 & 12.70 & 0.98 \\
\hline 7 & Fat mass ( kg ) & 9.55 & 5.85 & 0.73 \\
\hline 8 & Fat $(\%)$ & 11.87 & 5.75 & 0.13 \\
\hline 9 & Body Surface Area $\left(\mathrm{m}^{2}\right)$ & 1.94 & 0.21 & 0.87 \\
\hline
\end{tabular}

Table (1) results reveal that study sample characteristics follow the normal distribution, where skewness coefficient ranges between $(-0.07,1.87)$ i.e. between $( \pm 3)$, and this gives direct indication that the sample represents a normal distributed society and is free from non-normal distributions defects.

\section{Spatial domain:-}

Research measurements applied in two phases as follow:

\section{Phase I: Egyptian youth national team wrestlers:}

Body components and general physical measurements have been carried out at the laboratory of scientific management of measurements and tests at national teams' Olympic center 
in Cairo. while specific physical and skill measurements have been carried out at wrestling hall at national teams' Olympic center in Cairo.

Phase II: Wrestlers holding $1^{\text {st }}$ position (Alexandria clubs) .

Body components and general physical measurements have been carried out at the laboratory of physical fitness and sports for all at Faculty of Physical Education for Men, Alexandria University, while specific physical and skill measurements have been carried out at Faculty of Physical Education for Men, Alexandria University in same way as done in Olympic center, Cairo.

\section{Time domain:}

All measurements and tests have been carried out in the period from December $1^{\text {st }}$, to December $15^{\text {th }}, 2011$.

\section{Research Measurements and tests:}

\section{I - Basic measurements:}

- Age: calculated to the nearest half a month at beginning of measurement month (December $1^{\text {st }}, 2011$ )

- Total body height: - measured to the nearest centimeter.

- Body weight: - measured to the nearest kilogram.

- Body Surface Area:

Have been extracted using Dubois equation as follows:

Body surface Area $\left(\mathrm{m}^{2}\right)=$ weight $(\mathrm{kg})^{0.425} \mathrm{x}$ height $(\mathrm{cm})^{0.725}$ $\mathrm{x} 0.00718$ and matching the measurement using Nomogram chart

\section{II- Body component (BC): -}

- The body component measurements have been done using Tanita TBF - 300, Body Composition analyzer, which is one of the latest devices used to determine body components; body components measured are as follows:

- $\quad$ Fat-Free Mass (FFM)

- $\quad$ Body Mass Index (BMI)

- $\quad$ Total Body Water (TBW)
- $\quad$ Fat Mass (FM)

- $\quad$ Fat percentage $(\mathrm{F} \%)$

III- Specific skills measurements:

These measurements have been done using television filming to determine the correct number of attempts during the allotted time for each test

\section{- Power Endurance Test}

Back Cast Skill Performance Test (40 seconds) (Waleed Mohsen Nasra, 2004:p51)

\section{- Strength Endurance Test -}

Back Cast Skill Performance Test (1 minute) (Mohammad Reda Hafiz El-Roby, 2005: p214)

\section{- Specific Power Test}

Bridge Skill Performance Test (three times) (El Sayed Mohammed Issa, 1995:p 49)

\section{- Agility Test}

Bridge Skill Performance (10 seconds) (El Sayed Mohammed Issa, 1995:p 47)

\section{- Speed Endurance Test}

Bridge Skill Performance Test (30 seconds) (Mohammad Reda Hafiz El-Roby, 2005: p212)

\section{- Performance Endurance Test}

Bridge Skill Performance Test (60 seconds) (Mohammad Reda Hafiz El-Roby, 2005: p212)

Statistical factors and coefficients:-

- Mean

- Standard deviation

- $\quad$ Skewness coefficient

- Minimum value

- Maximum value

- correlation coefficient

Results:

Table (2)

Mean , standard deviation, minimum and maximum measurements for wrestlers body components

\begin{tabular}{|c|c|c|c|c|c|}
\hline No. & $\begin{array}{c}\text { Statistical significance } \\
\text { body components }\end{array}$ & Mean & $\begin{array}{c}\text { Standard } \\
\text { deviation }\end{array}$ & Minimum & Maximum \\
\hline 1 & Body mass index (\%) & 25.34 & 3.48 & 20.00 & 32.60 \\
\hline 2 & Total Body Water (TBW) (kg) & 49.83 & 8.03 & 38.70 & 67.60 \\
\hline 3 & Fat -free mass (FFM) (kg) & 69.97 & 12.70 & 52.80 & 99.20 \\
\hline 4 & Fat mass ( kg ) & 9.55 & 5.85 & 3.10 & 23.90 \\
\hline 5 & Fat (\%) & 11.87 & 5.75 & 4.70 & 25.80 \\
\hline 6 & Body Surface Area $\left(\mathrm{m}^{2}\right)$ & 0.21 & 1.60 & 2.30 \\
\hline
\end{tabular}


Table (2) results reveal that body components mean value ranged between while these variables minimum and maximum values were ( $3.10,52.80)$, and ( $23.90,99.20)$ respectively.

$(9.55,69.97)$ for fat mass and fat-free mass respectively

Figure 1

Minimum and maximum body components measurements for research sample
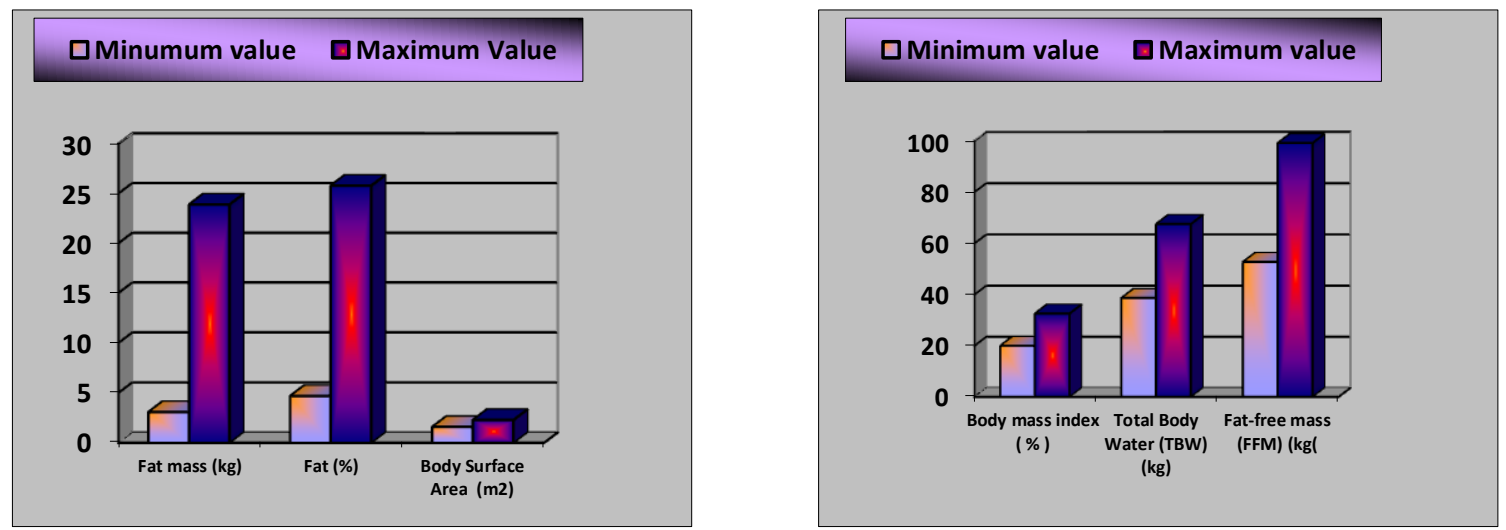

Table ( 3)

Mean, standard deviation, minimum and maximum measurements for wrestlers skills performance

\begin{tabular}{|c|c|c|c|c|c|}
\hline No & Statistical significance & Mean & $\begin{array}{c}\text { Standard } \\
\text { deviation }\end{array}$ & Minimum & Maximum \\
\hline 1 & Back Cast Skill Performance Test (40 seconds) & 16.61 & 1.85 & 12.00 & 21.00 \\
\hline 2 & Back Cast Skill Performance Test (1 minute) & 22.07 & 2.21 & 15.00 & 26.00 \\
\hline 3 & Bridge Skill Performance Test (three times) & 5.14 & 0.88 & 3.90 & 7.00 \\
\hline 4 & Bridge Skill Performance (10 seconds) & 6.11 & 0.99 & 4.00 & 8.00 \\
\hline 5 & Bridge Skill Performance Test (30 seconds) & 15.07 & 2.81 & 11.00 & 21.00 \\
\hline 6 & Bridge Skill Performance Test (60 seconds) & 24.93 & 3.29 & 18.00 & 31.00 \\
\hline
\end{tabular}

Table ( 3 ) results reveal that skills performance mean value ranged between $(5.14,24.93)$ for Bridge Skill Performance Test (three times)and Bridge Skill Performance Test (60 seconds)respectively while these variables minimum and maximum values were $(3.90,18.00)$, and $(7.00,31.00)$ respectively.

Figure 2

Minimum and maximum body components measurements for research sample

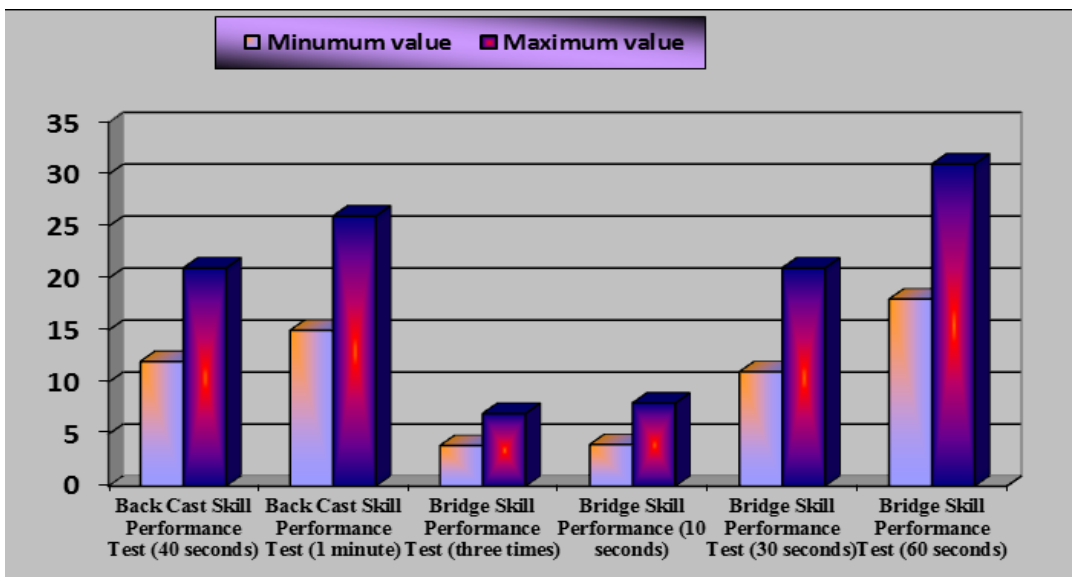


Table (4)

Correlation between wrestlers' skills performance level and physical components

\begin{tabular}{|c|c|c|c|c|c|c|}
\hline Body component & $\begin{array}{c}\text { Body } \\
\text { mass } \\
\text { Index } \\
(\%)\end{array}$ & $\begin{array}{c}\text { Total } \\
\text { Body } \\
\text { Water } \\
(\mathrm{TBW}) \\
(\mathrm{kg})\end{array}$ & $\begin{array}{c}\text { Fat -free } \\
\text { mass } \\
(\mathrm{FFM}) \\
(\mathrm{kg})\end{array}$ & $\begin{array}{c}\text { Fat } \\
\text { mass } \\
(\mathrm{kg})\end{array}$ & $\begin{array}{c}\text { Fat } \\
(\%)\end{array}$ & $\begin{array}{c}\text { Body } \\
\text { Surface } \\
\text { Area }\left(\mathrm{m}^{2}\right)\end{array}$ \\
\hline Back Cast Skill Performance Test (40 seconds) & -0.073 & -0.053 & 0.323 & -0.094 & -0.247 & -0.351 \\
\hline Back Cast Skill Performance Test (1 minute) & -0.165 & -0.152 & $0.491^{* *}$ & -0.295 & -0.33 & $-0.540^{* *}$ \\
\hline Bridge Skill Performance Test (three times) & -0.017 & -0.085 & $0.407 *$ & -0.323 & -0.289 & $-0.529 * *$ \\
\hline Bridge Skill Performance (10 seconds) & -0.154 & -0.068 & 0.055 & 0.147 & -0.172 & -0.167 \\
\hline Bridge Skill Performance Test (30 seconds) & -0.068 & -0.028 & 0.11 & 0.239 & -0.055 & 0.004 \\
\hline Bridge Skill Performance Test (60 seconds) & -0.105 & -0.133 & 0.287 & -0.213 & -0.229 & $-0.434 *$ \\
\hline
\end{tabular}

Table (4) results reveal presence of correlation between skills performance level and body components at (0.05),
(0.01) significance level, correlation coefficients ranged between $(0.407)$ and $(0.950)$

Table 5

simple correlation matrix between wrestlers' skill performance level and body components

\begin{tabular}{|c|c|c|c|c|c|c|c|c|c|c|c|c|}
\hline $\begin{array}{l}\text { Correlation } \\
\text { matrix }\end{array}$ & 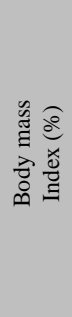 & 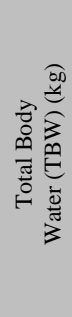 & 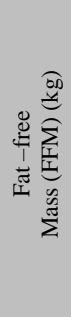 & 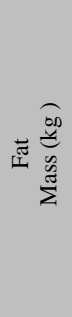 & 离 & 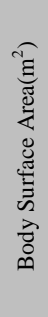 & 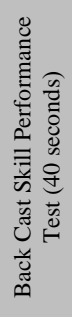 & 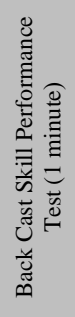 & 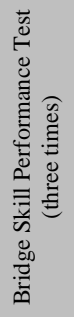 & 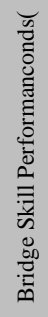 & 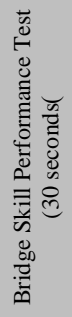 & 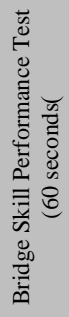 \\
\hline $\begin{array}{l}\text { Body mass index } \\
\qquad(\%)\end{array}$ & & $\begin{array}{l}\widetilde{\infty} \\
\infty \\
0\end{array}$ & $\begin{array}{l}\stackrel{*}{*} \\
\stackrel{*}{*} \\
\stackrel{\infty}{0} \\
\dot{0}\end{array}$ & $\begin{array}{l}\stackrel{*}{*} \\
\stackrel{*}{*} \\
\stackrel{\infty}{\infty} \\
0\end{array}$ & 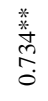 & 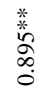 & $\stackrel{0}{0}$ & $\hat{\delta}$ & त̂र & $\begin{array}{l}+ \\
\text { Oे } \\
\dot{i}\end{array}$ & $\begin{array}{l}\text { I } \\
\text { i } \\
\text { i }\end{array}$ & $\begin{array}{l}\vec{n} \\
\substack{i \\
i}\end{array}$ \\
\hline $\begin{array}{c}\text { Total Body } \\
\text { Water (TBW) } \\
(\mathrm{kg})\end{array}$ & & & 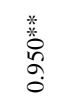 & $\begin{array}{l}\text { 类 } \\
0 \\
n \\
0\end{array}$ & 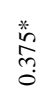 & 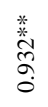 & 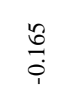 & $\frac{\pi}{2}$ & 挡 & $\begin{array}{l}\text { ते } \\
\text { ì }\end{array}$ & $\stackrel{m}{m}$ & 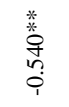 \\
\hline $\begin{array}{l}\text { Fat -free mass } \\
(\mathrm{FFM})(\mathrm{kg})\end{array}$ & & & & 誉 & तี & 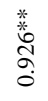 & $\begin{array}{l}\text { जo } \\
\stackrel{0}{i}\end{array}$ & $\stackrel{n}{0}$ & 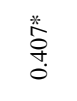 & 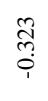 & $\begin{array}{l}\text { aे } \\
\stackrel{i}{1} \\
\text { i }\end{array}$ & 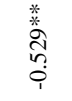 \\
\hline Fat mass ( $\mathrm{kg}$ ) & & & & & 葢 & 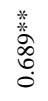 & 孛 & $\stackrel{\infty}{\circ}$ & $\underset{n}{n}$ & $\stackrel{\text { 守 }}{0}$ & $\underset{⿱}{\mathbb{1}}$ & $\begin{array}{l}\frac{5}{0} \\
\\
\end{array}$ \\
\hline Fat $(\%)$ & & & & & & 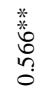 & $\begin{array}{l}\infty \\
\stackrel{\infty}{0} \\
\stackrel{i}{i}\end{array}$ & $\stackrel{\infty}{0}$ & $\stackrel{9}{7}$ & तुे & 盀 & 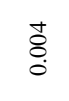 \\
\hline $\begin{array}{l}\text { Body Surface } \\
\text { Area }\left(\mathrm{m}^{2}\right)\end{array}$ & & & & & & & $\frac{\mho}{0}$ & $\stackrel{m}{\stackrel{m}{i}}$ & ্ָ & $\stackrel{m}{\stackrel{m}{9}}$ & $\begin{array}{l}\text { సे } \\
\text { ़ฺ }\end{array}$ & 烍 \\
\hline $\begin{array}{l}\text { Back Cast Skill } \\
\text { Performance Test } \\
\text { (40 seconds) }\end{array}$ & & & & & & & & \begin{tabular}{l}
$*$ \\
\multirow{2}{*}{} \\
$\stackrel{*}{\infty}$ \\
0 \\
0
\end{tabular} & 㐫 & ¿̊. & $\frac{\mathrm{S}}{0}$ & $\stackrel{n}{\varrho}$ \\
\hline $\begin{array}{l}\text { Back Cast Skill } \\
\text { Performance Test } \\
\text { (1 minute) }\end{array}$ & & & & & & & & & 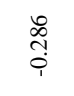 & $\stackrel{9}{\circ}$ & $\stackrel{\infty}{\stackrel{\infty}{!}}$ & $\stackrel{2}{0}$ \\
\hline
\end{tabular}




\begin{tabular}{|c|c|c|c|c|c|c|c|c|c|c|c|c|}
\hline $\begin{array}{c}\text { Correlation } \\
\text { matrix }\end{array}$ & 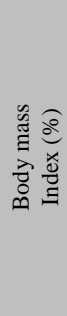 & 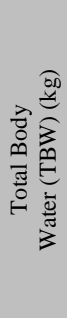 & 惫 & 死 & 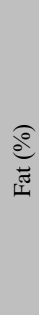 & 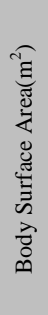 & 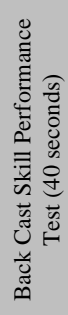 & 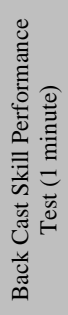 & 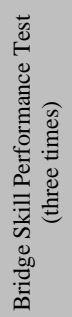 & 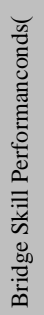 & 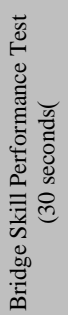 & 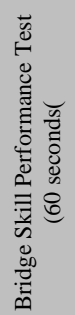 \\
\hline $\begin{array}{l}\text { Bridge Skill } \\
\text { Performance Test } \\
\text { (three times) }\end{array}$ & & & & & & & & & & $\begin{array}{l}\stackrel{*}{*} \\
\infty \\
\infty \\
? \\
i\end{array}$ & 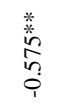 & $\begin{array}{l}\stackrel{*}{*} \\
\stackrel{*}{\square} \\
\stackrel{1}{i}\end{array}$ \\
\hline $\begin{array}{l}\text { Bridge Skill } \\
\text { Performance (10 } \\
\text { seconds) }\end{array}$ & & & & & & & & & & & $\stackrel{\text { * }}{\stackrel{f}{f}}$ & 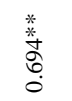 \\
\hline $\begin{array}{c}\text { Bridge Skill } \\
\text { Performance Test } \\
\text { (30 seconds) }\end{array}$ & & & & & & & & & & & & \begin{tabular}{l}
$*$ \\
\multirow{2}{*}{} \\
సे \\
0 \\
0
\end{tabular} \\
\hline $\begin{array}{l}\text { Bridge Skill } \\
\text { Performance Test } \\
(60 \text { seconds })\end{array}$ & & & & & & & & & & & & \\
\hline
\end{tabular}

Table (5) results reveal presence of correlation between skills performance level and body components at (0.05), (0.01) significance level, correlation coefficients ranged between (0.375) and (0.950)

\section{Discussion:}

With respect to tables (2), (3 ), results which describes mean, standard deviation, minimum and maximum values, it make clear importance of these variables as important indicators to selection process and prediction for junior could accomplish from results and achievements in practiced sports activity.

These indicators are considered a guide to read success indicators in training process and this is confirmed by scientific studies consensus about the importance of body components. Adel Abdel Halim Haider $(1999,54)$ and Zaky Mohammed Hassan (2004, p48) , argued that body components are of most important foundations for ensuring juniors' selection process success and directing the to various activities commensurate with them, it is possible also o use this indicators to follow up the evolution in training process and level development in general, they are a great help for coaches when making selection, which could distinguish their efforts to achieve economy in time and effort.

Bob Davis (1997, p279), Marusak , and Lenore, (1995, p12) emphasize that e individual's fitness for various sports is determined by the suitability of his body composition to perform the work required and in efficiency which body members can achieve movement aim. They indicated that body components have an effective role on physical and motor variables related the skill performance, and also affect the player motor coordination and abilities, in addition to contribution in sports training process development with what suit individual differences and choosing sport activity type and direct the junior to various sport activities that suit them according to each activity requirements.
Christopher (1995, p115) in the opinion that body's composition contains total weight of various tissues including bones, fat, fluids and different types of connective tissues, body's building and composition is important to the degree that it could be argued that it should be an adequate selection of the player in terms of his body composition to achieve success in global competitions.

Ali Fahmi El-Bek et al (2002, p44) indicate that determining body components, shape and characteristics directly contributes more than other characteristics in determining ability to achieve high levels in specific physical activity.

Tables ( 4), (5) (correlation matrix) results reveal presence of correlation between most variables, and between variables and skills performance level, some correlation are significant and the others are not significant, some are negative and some are positive .

In this regard, Mohammed Ibrahim El-Melegy (1998, p98), quoting from Pall G. Hwel that it is frequently happen to examine relationship between two variables or more, and then use the relationship derived in helping to estimate, direct or predict the values of one of the variable .

This is consistent with results of Mohammed Masoud et al ( 2012) Ahmed Salama and Ayman Abdul Aziz (2008), Selma Arzu et al(2007), Matthew Beekley et. al. (2006), Nader Shalaby (2002), Taha Al-Jasser and Ahmad Abu ElLeel (2002) which indicated presence of relationship between body components and skills performance level.

\section{Conclusions:}

As per research results and statistical work and limited by research sample researcher could arrive to:

1. Determine body components of top level wrestlers.

2. Determine skill performance of top level wrestlers 
3. That there is correlation between wrestlers' skills performance and body components

\section{Recommendations:}

Based on research conclusions and in light of its procedures researcher recommends the following:

1- Taking into account general specifications of body components concluded by the study for top level wrestlers in selecting suitable juniors for wrestling sport.

2- Taking into account skills performance concluded by the study for top level wrestlers in selecting suitable juniors for wrestling sport.

3- Taking into account the correlation between wrestlers' high skills performance and body components as an indicator of tune and standardize training loads .

4- Further research and scientific studies to be carried out regarding body and physiological components and their relation with skill performance in sports generally and wrestling specially, and compare results with top level global teams and then het practical and scientific advantage from these comparison.

5- Work to increase wrestling prevalence in clubs, and youth centers and provide the necessary support for measuring body components to increase selection area and expand practice base.

\section{References:}

1. Abul-Ela Abdel Fattah and Mohamed Sobhy Hassanein (1997) : Sports Physiology and morphology and measurement and evaluation methods, Dar Al-Fikr Al Arabi, Cairo .(in Arabic)

2. Ahmed Salam Shehata and Ayman Abdul Aziz Abdul-Hamid (2008) : Skill performance and its relationship with body measurements and play positions within basketball players , Journal of theories and applications , Faculty of Physical Education for Men- Alexandria University, No. 66 . (in Arabic)

3. El Sayed Mohammed Issa (1995) : Impact of proposed training program for the developing agility and power on skills performance for some back stepping groups within wrestlers, Ph.D. thesis , Faculty of Physical Education for Men, Alexandria University . (in Arabic)

4. Hussein Ahmed Heshmat (1999) : Biotechnology, Biochemical techniques and its applications in sports , University publishing house, Cairo.(in Arabic)

5. Taha Abdul Rahman Al-Jasser, Ahmad Ali Abu Elleel (2002):Body characteristics and components of football national team and first-class players in Kuwait as selection approach, theories and applications - Faculty of Physical Education for Men, Alexandria University, No. 45 (in Arabic)

6. Adel Abdul Halim Haider (1999) : anthropometric indicators of Egyptian basketball national team players , " factorial study ", Scientific journal of Physical Education and Sports , No. 16, Faculty of
Physical Education for Girls , Alexandria University (in Arabic)

7. Ali Fahmy El-Bek, Abul-Ela Abdel Fattah and Lotfi Kalini (2002) : Comparison of some morph functional changes within top level, athletics group athletics, First Scientific Conference: sports talents selection and preparation strategies in light of technological development and information revolution, first part, from October $30^{\text {th }}$ to November $1^{\text {st }}$, Faculty of Physical Education for Men, Alexandria University. (in Arabic)

8. Zaki Mohammed Hassan (2004) : morphological phenomena in team sports, Egyptian Library for publishing and distribution (in Arabic)

9. Mohammed Ibrahim Al-Melegy (1998) : Directing some biomechanical indicators to improve extraversion movement performance in fencing, $\mathrm{Ph} . \mathrm{D}$. thesis , Faculty of Physical Education for Men, Zagazig University. (in Arabic)

10. Mohammad Reda Hafiz El-Roby (2005) : Freestyle wrestling training principles, $1^{\text {st }}$ ed. , Mahy Publishing, Alexandria (in Arabic)

11. Mohammed Masoud Sharf, Waleed Mohsen Nasra and Walid Suleiman El-Saidi (2012) : Morphological specification and body within Judo top levels, Journal of theories and applications, Faculty of Physical Education for Men, Alexandria University, Issue 74, July.(in Arabic)

12. Nadir Mohamed Shalaby (2002) : Study of some body components of football, basketball and boxing national teams, theories and applications, $5^{\text {th }}$ issue for International Scientific : sports talents selection and preparation strategies in light of technological development and information revolution, Faculty of Physical Education for Men, Alexandria University (in Arabic)

13. Waleed Mohsen Nasra (2004) : Impact of back cast skill power endurance development on some skill and physiological variables within wrestlers, Master Thesis, Faculty of Physical Education for Men, Alexandria University (in Arabic)

14. Bob. Davis (1997): Physical Education and the Study of sport pub Mosby, London,

15. Christopher, M.N (1995): Weight Training Principles, A \& Black publisher, London

16. Horswill, P., (2008):The relationship between body composition and anaerobic performance of elite young wrestlers, fitness and nutrition, Vol. 4,

17. Janet L. Walberg, (1999): Weight Control and the Female volleyball player, Human Kinetics,

18. Marusak, Lenore (1995): Relationship between Morphological physical Fitness \&Motor Ability Measure to USA, Sec-Ram Kings in Female Junior Tennis players, California State University, Long Beach

19. Matthew Beekley et. al. (2006):Comparison of normalized maximum aerobic capacity and body composition of sumo wrestling to athletes in combat and other sports. Journal of sports science \&Medicine 5(CSSI), 13-20 USA

20. Oppliger, R. et al., (2008) :Weight loss in wrestlers, American college of sports medicine, Vol. 28, No.2

21. Selma Arzu et. al. (2007): The relationship between body composition and anaerobic performance of elite young wrestler Journal of sports science \&Medicine 6(CSSI), 34-38 Turkey 
